\title{
Routine interferon-neutralising antibody testing in patients with relapsing-remitting multiple sclerosis
}

\author{
${ }^{1} \mathrm{P}$ Foley, ${ }^{2} \mathrm{P}$ Reilly, ${ }^{3} \mathrm{~A}$ Coulson, ${ }^{4} \mathrm{JI}$ O'Riordan \\ ${ }^{1}$ Neurology Registrar; ${ }^{2}$ Neurology Specialist Nurse; ${ }^{3}$ Neurology Pharmacist; ${ }^{4}$ Consultant Neurologist, Ninewells Hospital, Dundee, UK
}

\begin{abstract}
Multiple sclerosis is a leading cause of disability in young adults, with the Scottish population suffering the highest prevalence in Europe. Disease-modifying therapies, including beta-interferon (IFN- $\beta$ ), are increasingly used to minimise relapse frequency in the majority of patients who present with a relapsing-remitting disease pattern. Unfortunately, neutralising antibodies (NABs) may develop against IFN- $\beta$ and are associated with reduced efficacy. These antibodies may be detected using a serum sample. Despite the importance of this problem, from both a patient's perspective and a wider community and economic standpoint, there is no universally agreed protocol for the use of NAB testing. Authorities variously suggest routine 'screening' testing or, conversely, testing only in specific situations. In Scotland, routine testing is seldom used. We report our experience of routine NAB testing in 105 patients (of whom 35 were NAB-positive) over two years in NHS Tayside and comment on its cost and implications.
\end{abstract}

KEYWORDS Interferon, multiple sclerosis, neutralising antibody

DECLARATION OF INTERESTS Dr O'Riordan has been a principal investigator in clinical trials sponsored by Merck Serono, Teva and Bayer Schering. He has also been a consultant to, or lectured at meetings sponsored by, Merck Serono, Teva, Bayer Schering and Biogen Idec. NHS Tayside is currently participating in the Department of Health's Risk Sharing Scheme on Disease Modifying Therapies in Multiple Sclerosis.
Correspondence to Jl O'Riordan, Tayside Regional MS Unit, Ninewells Hospital, Dundee DDI 9SY, UK

tel. $+44(0) 13826601$ II

e-mail joriordan@nhs.net

\section{INTRODUCTION}

Multiple sclerosis (MS) is a leading cause of disability in young adults in the Western world. It places an appreciable burden on the individual, as well as on families, carers and the health services. Prevalence in Scotland is high, and is thought to vary between 150 per 100,000 population in the west of Scotland to more than 250 per 100,000 in Orkney. In 80-85\% of individuals with MS, the disease first presents in a relapsing-remitting pattern, with periods of neurological symptoms or disability interspersed with periods of relative normality.

Disease-modifying therapies (DMTs) are increasingly widely used in an effort to reduce the frequency of MS relapses in the relapsing-remitting stage of the disease. These agents act to reduce the severity and frequency of relapses by around one third.'

Our practice is to use either beta-interferon (of which four preparations were available at the time of our study) or glatiramer acetate as first-line therapy, with natalizumab and mitoxantrone considered as potential second-line therapies for aggressive relapsing-remitting $\mathrm{MS}$ and relapsing-progressive MS, respectively.
Interferon therapies are, however, potentially immunogenic, and host antibodies may arise against the agent. These are known to be associated with reduced in-vivo efficacy of interferon, and are termed neutralising antibodies (NABs). Neutralising antibodies bind to the beta-interferon (IFN- $\beta$ ) molecule and cause disruption of the normal biological action of the drug by affecting binding to its receptor. ${ }^{2}$ Antibodies against glatiramer acetate are not felt to be of clinical relevance and are not further considered here. ${ }^{3}$

There is no universally agreed standard method for $N A B$ testing. However, in general, the first step is to screen for antibodies binding to IFN- $\beta$, generally by enzyme-linked immunosorbent assay (ELISA). A further assay, specifically for neutralising activity, is then carried out, seeking suppression of the in-vivo action of IFN- $\beta$. The most commonly used assay is the cytopathic effect assay in which the ability of IFN- $\beta$ to inhibit viral replication in culture cells is measured..$^{2,4}$

A variable proportion of patients may become NABpositive. Neutralising antibodies are not thought to be associated with systemic side effects; however, there is robust data to suggest that the development of NAB positivity is associated with the decreased clinical 
P Foley, P Reilly, A Coulson, JI O’Riordan

effectiveness of IFN- $\beta$, both in decreasing relapse rate and modifying brain magnetic resonance imaging (MRI) outcomes.

Neutralising antibody positivity is by no means the only factor associated with reduced clinical efficacy of interferon, and it is not certain that NAB positivity is a causative factor. Several randomised controlled trials of interferon (including major landmark trials of both IFN- $\beta$-IB and IFN- $\beta$-IA) have, however, demonstrated a reduction in efficacy on relapses in patients who developed NAB positivity. ${ }^{5.6}$ In a major phase three trial of IFN- $\beta$-IB, relapse rates in $\mathrm{NAB}$-positive patients were equivalent to relapse rates in those given placebo. ${ }^{6}$ In a prospective Danish study, NAB-positive patients were found to have a significantly shorter time to first relapse, with the proportion of patients free of relapse being significantly lower. ${ }^{7}$

Similarly, MRI outcomes (such as the number of inflammatory lesions seen) are adversely affected by NAB positivity, with more active MRI lesions observed in patients who are NAB-positive. ${ }^{5.6}$ In one study NABpositive patients had almost five times the median number of active lesions on T2 sequences compared with NAB-negative patients. ${ }^{5}$

There is, however, no universal agreement on the optimal system for $N A B$ testing and, in particular, no agreement on whether $N A B$ testing should be carried out routinely in all patients. Suggestions made by different authorities vary. The European Federation of Neurological Sciences (EFNS) consensus guidelines (2005) recommend routine NAB testing at 12 and 24 months after starting interferon therapy, with further action dictated by titre result (in other words the strength of NAB activity) ${ }^{8}$ It is suggested that a high titre which is sustained at repeated testing should prompt discontinuation of interferon therapy. The Association of British Neurologists (ABN), however, stop short of such a recommendation and suggest that, in the case of clinical or radiological deterioration, positive NABs, especially if at high titre and sustained, only strengthen a case for discontinuation of interferon therapy. ${ }^{9}$ Thus one authority suggests routine testing of NABs in all relevant patients, and another suggests only testing when clinical or radiological deterioration occurs. Usual practice in the UK in general, and Scotland specifically, favours the latter approach, with NABs only tested in the context of a clinical deterioration.

In contrast, our practice is to routinely screen for $N A B$ positivity in all patients receiving interferon, following a local protocol based on current EFNS guidelines. We believe that routine NAB testing is a valuable adjunct to the decision-making process when deciding on appropriate DMT for any patient on interferon. We report here our experience of two years of routine NAB testing in NHS Tayside.

\section{METHODS}

All patients receiving IFN- $\beta$ between April 2007 and April 2009 were enrolled in our audit. We recorded details of patients undergoing $N A B$ testing, the preparation of interferon received, $N A B$ titre results, results of repeat testing (where applicable) and treatment decisions made. Patients were receiving one of four IFN- $\beta$ preparations: interferon $\beta$-IB ('Betaferon', Bayer) or interferon $\beta$-IA ('Rebif $22 \mu \mathrm{g}$ ' dosing or 'Rebif $44 \mu \mathrm{g}$ ' dosing, both Merck-Serono, or 'Avonex', Biogen). Our experience agrees with data accrued in major clinical trials (including those comparing glatiramer acetate with Rebif and with Betaferon) in suggesting that clinical and MRI efficacy of these agents is comparable. ${ }^{10}$ When using Rebif we generally use the $22 \mu \mathrm{g}$ preparation initially, with local experience suggesting that the $22 \mu \mathrm{g}$ preparation is better tolerated by patients with, in particular, less local cutaneous reactions. None of the patients at the time of audit were prescribed new formulation Rebif, which has since become available.

All patients were attending the NHS Tayside subspecialist DMT clinic at Ninewells Hospital, Dundee. Testing was performed at 12 and 24 months after starting therapy, or at 12 and 24 months after commencement of NAB testing in patients who had already been established on therapy. Patients were made aware of testing, which was carried out as part of routine clinical care. Venous blood samples were taken in a clotted tube at the time of the patient's routine clinic review (along with other routine monitoring). Blood samples were sent to the Neuroimmunology Unit, Institute of Neurology, Queen Square, London, for analysis. This unit provides centralised NAB analysis for centres throughout the UK.

In patients with a high positive titre (>100 neutralising units) or with a positive titre at a lower level, change in treatment was considered on an individual basis.

In patients taking any IFN- $\beta$, change of therapy to copaxone, natalizumab or mitoxantrone, as well as the cessation of therapy, were considered. We also considered changing patients from one interferon preparation to another. We took into account that NABs are felt to be cross-reactive and thus changing from one IFN- $\beta$ preparation to another may not avoid clinically significant reduction of efficacy related to NABs. None of the patients included in this audit commenced natalizumab ('Tysabri') monoclonal antibody therapy.

Costs of NAB testing were provided by the NHS Tayside finance department. Costs of drug therapy were provided by the neurology pharmacist. Mathematical analyses of costs and savings were carried out using Microsoft Excel. 


\section{RESULTS}

A total of 105 patients were tested. In the two-year period of testing, 35 patients returned positive NAB titres on initial analysis, with a range of titres between 20 and 640 neutralising units (NU).

\section{Patient demographics}

Of the patients tested, duration of drug therapy at the time of testing was as follows:

- I year - 2 patients

- 2 years - II patients

- 3 years - 12 patients

- 4 years -35 patients

- 5 years -24 patients

- 6 years - 10 patients

- 7 years - 10 patients

- 9 years - I patient

Of the 105 patients tested, 84 had relapsing-remitting MS, and $2 \mathrm{I}$ had secondary progressive MS with relapses.

\section{NAB titres}

Of the 35 positive titres, 24 were at high positive levels (>100 NU, range 120-640) and II were at low positive levels ( $\leq 100 \mathrm{NU}$, range 20-100). The cut-off for defining high positive titres is generally considered to be $100 \mathrm{NU}{ }^{8}$

Of those returning low positive titres, all were retested. Six of these reverted to negative titres (range of initial positive titre 20-100 NU). Repeat titres are awaited in one patient.

Of those patients demonstrating positive titres, very high titres (>100 NU) were not retested, and treatment was modified according to the judgement of the treating clinician. It is our experience - in keeping with other authors' findings - that in patients who manifest high titres, reversion to low titre status is uncommon. ${ }^{4}$
TABLE I Neutralising antibody test results and treatment decisions grouped by drug

\begin{tabular}{|l|l|l|l|l|}
\hline & $\begin{array}{l}\text { No. } \\
\text { tested }\end{array}$ & $\begin{array}{l}\text { Total } \\
\text { NAB- } \\
\text { positive }\end{array}$ & $\begin{array}{l}\text { Of which } \\
\text { >100 NU } \\
\text { (\% of } \\
\text { tested) }\end{array}$ & $\begin{array}{l}\text { Clinical } \\
\text { decisions }\end{array}$ \\
\hline Betaferon & 28 & 17 & $10(36)$ & $\begin{array}{l}\text { I changed to } \\
\text { copaxone; } \\
\text { 9 stopped } \\
\text { therapy }\end{array}$ \\
\hline Rebif 22 & 37 & 13 & $10(27)$ & $\begin{array}{l}\text { I0 changed } \\
\text { to copaxone }\end{array}$ \\
\hline Rebif 44 & 8 & 2 & 1 (13) & $\begin{array}{l}\text { I changed to } \\
\text { copaxone }\end{array}$ \\
\hline Avonex & 32 & 3 & $3(9)$ & $\begin{array}{l}\text { I changed to } \\
\text { copaxone; } \\
\text { I changed to } \\
\text { betaferon; } \\
\text { I changed to } \\
\text { mitoxantrone }\end{array}$ \\
\hline Total & 105 & 35 & 24 & $\begin{array}{l}\text { I3 changed to } \\
\text { copaxone; } \\
\text { I changed to } \\
\text { betaferon; } \\
\text { I changed to } \\
\text { mitoxantrone; } \\
\text { 9 stopped } \\
\text { therapy }\end{array}$ \\
\hline
\end{tabular}

Results obtained and clinical decisions made are outlined in Table I. All low positive titres returned either low positive or negative results on repeat.

\section{Frequency of NAB positivity at high titre}

The frequency of $\mathrm{NAB}$ positivity at a high titre of $>100 \mathrm{NU}$ in our population (as a percentage of those tested) was compared with previously published rates. ${ }^{5,6,11-15}$ The frequency of NAB positivity previously reported varies between different studies and is affected by, among

TABLE 2 Costs of IFN therapy and NAB testing, 2007-09*

\begin{tabular}{|l|l|l|l|l|l|l|}
\hline Drug & $\begin{array}{l}\text { Cost per } \\
\text { patient per } \\
\text { year }(\boldsymbol{\epsilon})\end{array}$ & $\begin{array}{l}\text { Number } \\
\text { of patients } \\
\text { tested }\end{array}$ & $\begin{array}{l}\text { Total IFN } \\
\text { cost per } \\
\text { year }(\boldsymbol{\epsilon})\end{array}$ & $\begin{array}{l}\text { Cost of } \\
\text { testing per } \\
\text { year }(\boldsymbol{\epsilon})\end{array}$ & $\begin{array}{l}\text { Number } \\
\text { changed or } \\
\text { stopped }\end{array}$ & $\begin{array}{l}\text { Financial implication per } \\
\text { year after subtracting } \\
\text { cost of testing ( } \boldsymbol{\epsilon})\end{array}$ \\
\hline Betaferon & 7,231 & 28 & 202,468 & 3,194 & 10 & $\begin{array}{l}63,317 \\
(\text { Net difference) }\end{array}$ \\
\hline Rebif 22 & 7,501 & 37 & 277,537 & 4,221 & 10 & $\begin{array}{l}12,808 \\
\text { (Net difference) }\end{array}$ \\
\hline Rebif 44 & 8,931 & 8 & 71,448 & 912 & 1 & $\begin{array}{l}2,220 \\
\text { (Net difference) }\end{array}$ \\
\hline Avonex & 8,502 & 32 & 272,064 & 3,650 & 3 & $\begin{array}{l}8,324 \\
(\text { Net difference) }\end{array}$ \\
\hline Total & & 105 & 823,517 & 11,977 & 24 & $\begin{array}{l}86,669 \\
(\text { Net difference) }\end{array}$ \\
\hline
\end{tabular}

*All costs given in Pounds Sterling, 2009. 


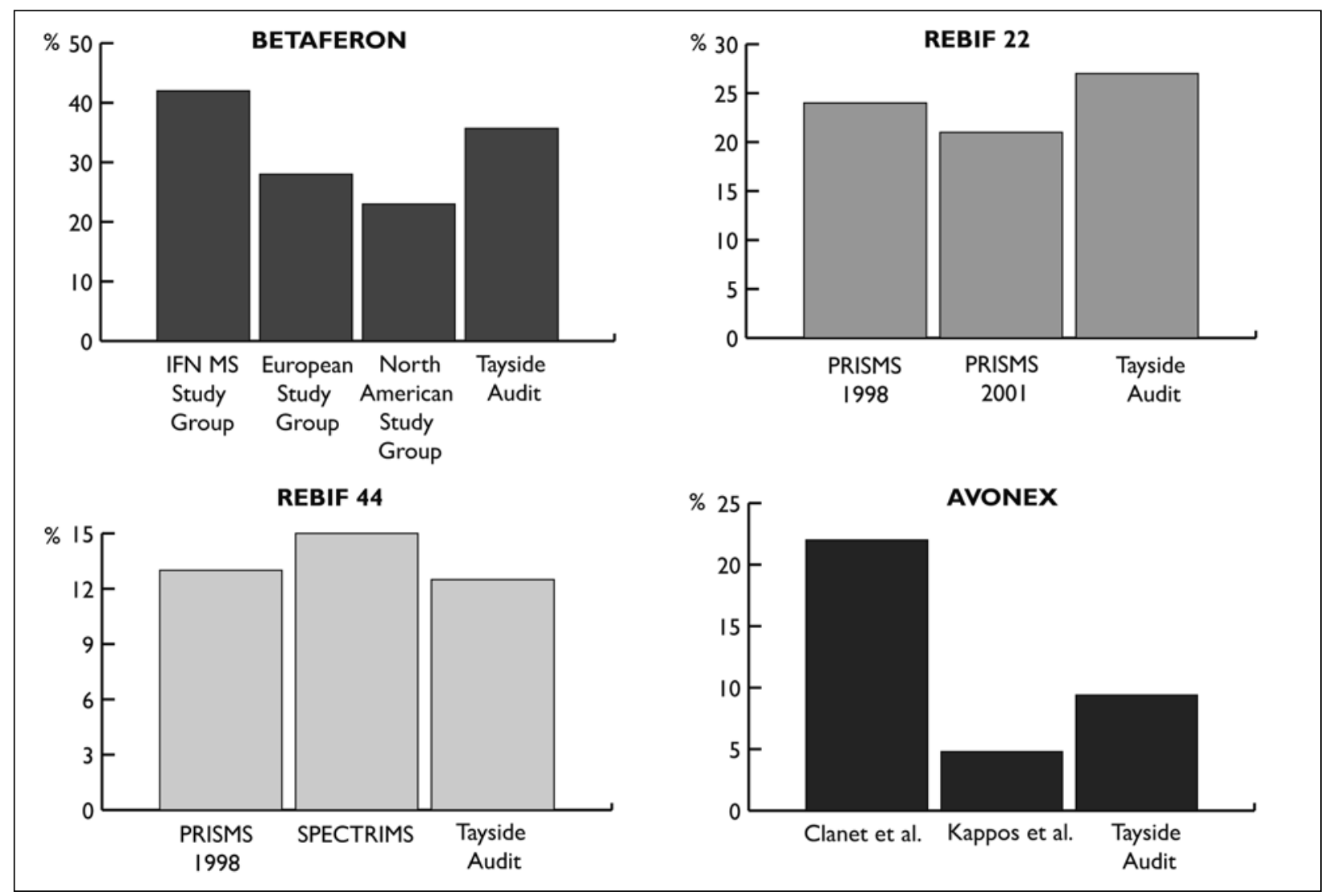

FIGURE I Frequency of high NAB positivity as a percentage of tests undertaken.

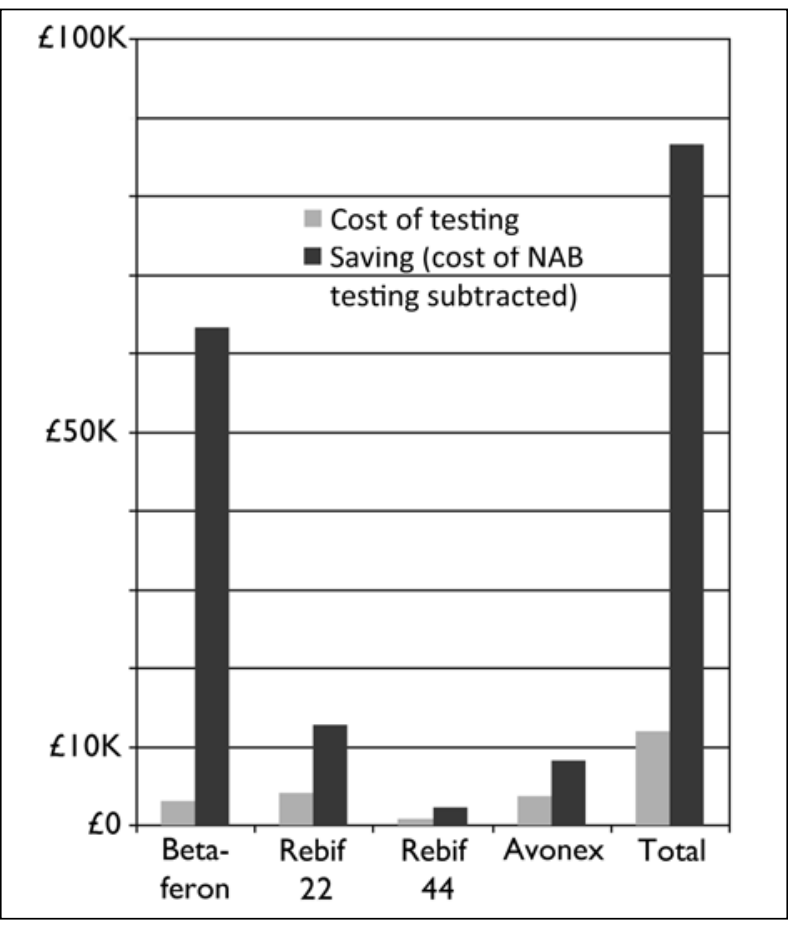

FIGURE 2 Cost of testing NAB titres compared with financial implications. other factors, the agent itself (including any changes in formulation), the route of administration, assay (including interassay variability) and patient characteristics. Despite this, our rates of NAB positivity were comparable to those previously published (Figure I).

\section{Financial implications of NAB testing}

Given that the direct cost of NAB testing has previously been felt to be a potential barrier to routine NAB testing, we were interested to estimate the financial implications of this approach. Financial costs of NAB testing (provided by NHS Tayside accountancy service) were $€ 7,808$ for the year April 2007-April 2008, and $\notin 4, I 7 \mid$ for the year 2008-2009. The mean cost of testing each patient was therefore $E I \mid 4.09$. The costs of interferon and copaxone treatment per patient year are given in Table 2.

We calculated for each drug the total cost of drug administration for all patients receiving the agent per year, the total cost of testing per year, and the number or patients in whom therapy was switched or stopped due to positive NAB titres. By comparing the cost of the previous and new agents and subtracting the cost of testing, we obtained estimated annual cost implications, which are summarised in Table 2. The net price difference per year directly compared with the cost of testing for each separate IFN drug is shown in Figure 2. 


\section{DISCUSSION}

Disease-modifying therapies are becoming more frequently used in the management of $M S$ - a disease which is a major cause of morbidity and disability in the UK in general and Scotland in particular. Beta-interferons and glatiramer acetate are used as first-line agents in an effort to decrease the frequency of MS relapses. Host NABs may arise against interferon and are associated with reduced efficacy; however, there is no universal agreement on the best strategy for testing NABs.

We feel that routine screening for NAB positivity may be a useful part of the assessment of MS patients in the UK, and can be used relatively easily with little inconvenience for the patient (particularly as blood tests are examined routinely at outpatient appointments). Weighed against this relatively minor burden is a potentially significant benefit.

Advocates of routine $N A B$ testing suggest that, since NABs are associated with reduced biological and therapeutic activity of interferons, IFN- $\beta$ should not be prescribed if NAB positivity develops. ${ }^{5}$ The patient may gain from this approach by avoiding unnecessary prolongation of interferon therapy and by expediting appropriate switching (or cessation) of therapy. This may confer benefits to the patient by minimising unnecessary pain and discomfort of drug therapy, minimising risk of side effects and, crucially, by ensuring that the patient is receiving the optimal therapy for his or her disease.

Possible burdens of routine NAB testing may include provoking anxiety in an otherwise stable patient if NABs are tested in the absence of clinical deterioration. Our experience has, however, been that patients are happy to proceed with routine NAB testing as long as the procedure is discussed before the time of testing.

The optimum strategy for use of NAB testing is complicated by questions including the extent of variability of $N A B s$ in a defined patient group, which may be linked to interassay variability. Available data, however, suggest that rate of conversion from NAB-negative to NAB-positive status (or vice versa) in a selected patient is uncommon, particularly at very low or very high titres of NABs. ${ }^{4}$ The relative utility and reliability of differing methods of NAB estimation (for instance by measuring antiviral effect using the cytopathic effect assay, as in this study; or by measuring IFN- $\beta$-induced gene products using the myxovirus resistance protein $A$ assay) also remain to be fully established. ${ }^{8}$

The expense of testing to the service concerned has been considered as a potential deterrent to routine testing. ${ }^{16}$ While, of course, we make the argument in favour of NAB testing on grounds of clinical utility and not of cost-effectiveness, our data suggest that the expense of testing should not represent a barrier to routine NAB testing. Further financial analysis is certainly warranted. The financial costs associated with routine NAB testing are, in our experience, offset by net savings attributable to appropriate changes in DMT (or cessation of therapy). Financial analysis (and in particular costeffectiveness analysis) would further be strengthened by clinical outcome data for each patient group and qualityadjusted life-year analysis.

A strength of our study lies in demonstrating the clinical feasibility of routine NAB testing in an outpatient setting which is comparable to many in the UK. In addition, an preliminary cost analysis suggests that costs are, at the least, not prohibitive.

\section{CONCLUSION}

While further questions remain (including, for example, the use of NAB testing compared or combined with routine $M R I$ imaging), ${ }^{17,18}$ we believe that routine NAB testing may be a valuable and cost-effective tool for neurology centres in the UK.

\section{REFERENCES}

I Compston A, Confavreux C, Lassmann H, editors. McAlpine's multiple sclerosis. 4th ed. Edinburgh: Churchill Livingstone; 2006.

2 Farrell R, Giovannoni G. Measuring and management of antiinterferon beta antibodies in subjects with multiple sclerosis. Mult Scler 2007; I2:567-77. doi:10.1 I77//352458506073522

3 Teitelbaum D, Brenner T, Abramsky $\mathrm{O}$ et al. Antibodies to glatiramer acetate do not interfere with its biological functions and therapeutic efficacy. Mult Scler 2003; 9:592-9. doi: I 0.I I 9I//352458503ms963oa

4 Sominanda A, Hillert J, Fogdell-Hahn A. Neutralizing antibodies against interferon beta: fluctuation is modest and titre dependent. Eur J Neurol 2009; 16:2 I-6. doi: I0. I I I I/j. I468- I33I.2008.02264.x

5 PRISMS Study Group and the University of British Columbia MS/ MRI Analysis Group. PRISMS-4: long-term efficacy of interferon beta- Ia in relapsing MS. Neurology 200I; 56:1628-36. Erratum in: Neurology 2001; 57:I 146.
6 The IFNB Multiple Sclerosis Study Group. Interferon beta-IB is effective in relapsing-remitting multiple sclerosis. I. Clinical results of a multicenter, randomized, double-blind, placebo-controlled trial. Neurology 1993; 43:655-6I.

7 Sorensen P, Ross C, Clemmensen KM et al Clinical importance of neutralising antibodies against interferon beta in patients with relapsing-remitting multiple sclerosis. Lancet 2003; 362:II84-9I. doi:I0.10I6/SO|40-6736(03) |454I-2

8 Sørensen PS, Deisenhammer F, Duda P et al. Guidelines on the use of anti-IFN-beta antibody measurements in multiple sclerosis: report of an EFNS Task Force on IFN-beta antibodies in multiple sclerosis. Eur J Neurol 2005; I2:8I7-27. doi: I0.I I I I/j.| I468-I33I.2005.01386.x

9 Association of British Neurologists (ABN). ABN Guidelines for treatment of multiple sclerosis with $\beta$-interferon and glatiramer acetate. London:ABN; 2007. 
I0 Mikol DD, Barkhof F, Chang P et al. Comparison of subcutaneous interferon beta-la with glatiramer acetate in patients with relapsing multiple sclerosis (the REbif vs Glatiramer Acetate in Relapsing MS Disease [REGARD] study): a multicentre, randomised, parallel, open-label trial. Lancet Neurol 2008; 7:903-|4. doi:I0.1016/ SI 474-4422(08)70200-X

II SPECTRIMS (Secondary Progressive Efficacy Clinical Trial of Recombinant Interferon-beta- Ia in MS) study group. Randomized controlled trial of interferon-beta- Ia in secondary progressive MS: clinical results. Neurology 200 I; 56:1496-504.

12 European Study Group on interferon beta-Ib in secondary progressive MS. Placebo-controlled multicentre randomised trial of interferon beta-Ib in treatment of secondary progressive multiple sclerosis. Lancet 1998; 352:149|-7.

13 The North American Study Group on interferon beta-Ib in Secondary Progressive MS. Interferon beta-lb in secondary progressive MS: results from a 3-year controlled study. Neurology 2004; 63: $1788-95$.
14 Clanet M, Kappos L, Hartung HP et al. Interferon beta I-a in relapsing multiple sclerosis: four-year extension of the European IFN beta-Ia dose-comparison study. Mult Scler 2004; 10:139-44. doi:I0.II9I/I352458504ms990oa

15 Kappos L, Clanet M, Sandberg-Wollheim M et al. Neutralizing antibodies and the efficacy of interferon beta-la - a 4-year controlled study. Neurology 2005; 65:40-7. doi:I0.1212/0I. wnl.0000I7I747.59767.5c

16 Burks JS, Noronha A. Guidelines on the use of anti-IFN-B antibody measurements in multiple sclerosis: report of an EFNS Task force on IFN-B antibodies in multiple sclerosis. Eur J Neurol 2007; |4:e8-9. doi:I0.1 I | I/j.|468-I33I.2007.0I782.x

17 Durelli L, Barbero P, Bergui M. MRI activity and neutralising antibodies as predictors of response to interferon beta treatment in multiple sclerosis.J Neurol Neurosurg Psychiatry 2008; 79:646-5I. doi:I0.II36/jnnp.2007.130229

18 Deisenhammer F. Neutralizing antibodies to interferon-beta and other immunological treatments for multiple sclerosis: prevalence and impact on outcomes. CNS Drugs 2009; 23:379-96. doi:10.2165/00023210-200923050-00003

\section{CARE OF THE ELDERLY SYMPOSIUM}

\section{Wednesday 15 September at the Royal College of Physicians of Edinburgh}

The population is ageing; healthcare professionals from different specialties are responsible for the care of an increasing number of older people. This symposium has been organised by 'jobbing' physicians with a major interest in the care of older people. The choice of topics has been guided by feedback from delegates at previous Care of the Elderly symposia.

Tele-healthcare in older people is an emerging area; the use of technology in the management of older people will be explored. Two speakers will address nutritional problems, both undernutrition and obesity, in older people. The final session of the day will be led by a surgeon, anaesthetist and geriatrician; it will centre on the key theme of multidisciplinary care in medicine of the elderly by investigating how to optimise surgical outcomes in older people.
Please see http://events.rcpe.ac.uk for further details and to book online or contact Christine Berwick (0I3I 247 3634, c.berwick@rcpe.ac.uk).

All health professionals involved in the care of older people are welcome.

\section{ADVANCE NOTICE UPDATE COURSE IN ELDERLY MEDICINE}

\section{Monday 16 - Friday 20 May 201 I}

\section{Course Director: Dr Andrew T Elder, FRCP Edin, Consultant Geriatrician}

This course is primarily designed for non-UK based consultants, specialists and senior trainees in elderly medicine from around the world. It offers a valuable opportunity to discuss clinical issues in elderly care. Places are limited to 60 so early application is advised. If doctors resident or working in the UK wish to apply, their details will be placed on a waiting list and they will be contacted in early January $201 \mathrm{I}$ to confirm whether or not a place is available.

Each day will focus on a single clinical topic:

- Preventing readmission of elderly people

- First do no harm

- Practical psychiatry for geriatricians

- Stroke disease

Mobility and function

\section{COURSE FEES:}

Weekly rate: $£ 720$ (inc.VAT) Daily rate: $£ 200$ (inc.VAT) Late registration fee: $£ 50$ if registering after 4 April $201 \mathrm{I}$.

For further details please see: http://events.rcpe.ac.uk or contact:

Christina Gray,

Education, Training and Standards Department, Royal College of Physicians, 9 Queen Street, Edinburgh EH2 IJQ, UK Tel: +44(0) I 3 I 2473607 Email: c.gray@rcpe.ac.uk 